\title{
Kinetics of internal structures growth in magnetic suspensions
}

\author{
G. Bossis ${ }^{\text {a }}$, P. Lançon ${ }^{\mathrm{a}}$, A. Meunier ${ }^{\mathrm{a}}$, L. Iskakova ${ }^{\mathrm{b}}$, V. Kostenko $^{\mathrm{b}}$, A. Zubarev ${ }^{\mathrm{b}, *}$ \\ ${ }^{a}$ Laboratoire de Physique de la Matière Condensée, CNRS UMR 7336, Université de Nice-Sophia Antipolis, Parc Valrose, 06108 Nice Cedex 2, France \\ ${ }^{\mathrm{b}}$ Urals Federal University, pr.Lenina 51, Ekaterinburg, Russia
}

\section{A R T I C L E I N F O}

\section{Article history:}

Received 24 May 2012

Received in revised form 25 October 2012

Available online 14 December 2012

\section{Keywords:}

Magnetic suspension

Aggregates

Kinetics, dipolar interactions

\begin{abstract}
A B S T R A C T
The kinetics of aggregation of non Brownian magnetizable particles in the presence of a magnetic field is studied both theoretically and by means of computer simulations. A theoretical approach is based on a system of Smoluchowski equations for the distribution function of the number of particles in linear chain-like aggregates. Results obtained in the two dimensional (2D) and three dimensional (3D) models are analyzed in relation with the size of the cell, containing the particles, and the particle volume fraction $\varphi$. The theoretical model reproduces the change of the aggregation kinetics with the size of the cell and with the particle volume fraction as long as the lateral aggregation of chains is negligible.

The simulations show that lateral aggregation takes place when, roughly, $\varphi_{2 \mathrm{D}}>5 \%$ and $\varphi_{3 \mathrm{D}}>1.5 \%$.

Dependence of the average size of the chains with time can be described by a power law; the corresponding exponent decreases with the particle volume fraction in relation with the lateral aggregation.

In the 3D simulations, dense labyrinthine-like structures, aligned along the applied field, are observed when the particle concentration is high enough $\left(\varphi_{3 \mathrm{D}}>5 \%\right)$.
\end{abstract}

(C) 2012 Elsevier B.V. All rights reserved.

\section{Introduction}

Suspensions of micron-sized magnetizable particles in non magnetic liquids (magnetorheological suspensions, MRS) attract considerable interest from researchers and engineers due to a rich set of unique physical properties, valuable for many modern technologies. An overview of the physics of these systems as well as their practical applications can be found in Ref. [1].

Without a magnetic field MRSs behave like ordinary suspensions of solid particles. When an MRS is subjected to an external magnetic field, the particles are magnetized and, under the action of the magnetic forces, form heterogeneous aggregates-linear chains and dense bulk clusters. The formation of these aggregates changes dramatically the macroscopical properties of MRS. For example, under an applied magnetic field the effective viscosity of MRS can increase up to several orders of magnitude. When the aggregates entirely fill the chamber (channel) containing the suspension and bond its opposite boundaries, the rheological behavior of the MRS changes from viscous to quasi elastic as long as the applied stress remains lower than the so called yield stress. Simultaneously the electrical conductivity of MRS increases significantly.

Practical applications of magnetic suspensions depend on the rate of change of their properties after application of the magnetic field. This is why the study of the growing rate of the aggregates is important for the prediction of the response time of MRS. To the best of our knowledge, the first model of the kinetics of evolution of the chain lengths in the systems of

\footnotetext{
* Corresponding author. Tel.: +7 3433507541.

E-mail address: andrey.zubarev@usu.ru (A.Zubarev).
} 
magnetizable particles has been developed by M. Doi et al. [2]. In this hierarchical theory all the aggregates have the same size at a given time, which is a quite crude approximation. Nevertheless this model takes into account that the force between two aggregates depends on their respective size and also that the average distance between aggregates increases during the aggregation process. Several numerical simulations have focused on the exponent of the growth of the chain average size $l$ with time: $l(t) \propto t^{\alpha}$ and have found $\alpha$ between 0.5 and 0.7 ; the last limit corresponds to the case where dipolar forces dominate Brownian forces [3,4]. A detailed analysis of the power law exponents was carried out both by Brownian dynamics simulation and experimentally, for large box sizes with energy of the dipole-dipole interaction of the particles being between two and three order of magnitude larger than the thermal energy $k T$ [5]. No clear evolution was seen in this range of dipolar forces; on the other hand the exponent $\alpha$ increased with the volume fraction. The analysis of the continuum version of the Smoluchowski equations in the presence of the dipolar forces has been investigated in Ref. [6] with a chain diffusion coefficient inversely proportional to the length of the chains; some analytical form for the size distribution function was obtained in this work. A model of aggregation of Brownian magnetic particles in linear chains has been developed in Ref. [7] on the basis of the Smoluchowski equations for the distribution function of the number of particles in the chain. The results of this model are in good agreement with experiments. Nevertheless the theoretical results were determined through the assumption that the Brownian motion strongly affects the kinetics of the chain formation. At the same time, in many cases the effect of Brownian motion in MRS is negligible because the energy of magnetic interaction between the micron-sized particles is usually several orders of magnitude larger than the thermal energy $k T$.

Recently an analytical model of the kinetics of the chain formation in a system of magnetizable non Brownian particles has been developed. [8]. This model is based on the usual system of the Smoluchowski equations for the density of chains of a given length. The size distribution functions obtained from the numerical solution of these equations reproduces well the distribution obtained from the computer simulations.

In this work we present results of computer simulations and of analytical modeling of aggregation in MRS. Both, 2D and 3D systems are considered. The effect of the size of the cell, containing the MRS, on the aggregation kinetics is studied.

The organization of this work is the following: in the next part we present the main features of the analytical model. In Section 3 we present the model used for the computer simulations. The comparison between the analytical and computer results is presented in Section 4 for 2D simulations and in Section 5 for 3D simulations. Computer simulations show that the particles form linear chains when their volume concentration is low enough (about $1 \%-2 \%$ ). When the concentration of the particles exceeds some threshold magnitude, dense bulk structures appear instead of the linear chains. The evolution of this system of thick clusters is studied in Section 6

\section{Analytical model of the chain growth}

The details of the analytical model were described in Ref. [8]. Here we will briefly recall the main points of this model. We consider a suspension of non Brownian magnetizable particles in a flat gap. The system is subjected to a homogeneous magnetic field $\mathrm{H}$ perpendicular to the gap boundary. We suppose that, under the field action, the particles coalesce uniquely in linear chains aligned along the field. It should be stressed that the appearance of any branched or bulk aggregates is ignored in this model. That is why it is restricted to suspensions of relatively low volume fraction.

Let us denote the number of $n$-particle chains per unit volume of the system as $g_{n}$. Our aim is to determine the evolution of this function with time.

In the framework of the Smoluchowski equations, the evolution of the chain population can be represented by the following system of kinetics equations:

$$
\frac{\mathrm{d} g_{n}}{\mathrm{~d} t}=\frac{1}{2} \sum_{k=1}^{n-1} \alpha_{n-k, k} g_{n-k} g_{k}-g_{n} \sum_{l=1}^{N-n} \alpha_{n, l} g_{l} .
$$

Here $\alpha_{n l}$-is a matrix of kinetics coefficient for the transformation of two chains of $n$ - and $l$-particles into a chain with $(n+l)$ particles, $N$ is the maximal number of particles that a chain can contain; $N$ is determined by the gap thickness. According to their physical meaning, the coefficient $\alpha_{n l}$ must be symmetrical with respect to the indexes $n$ and $l$. Taking this into account one can show that Eq. (1) automatically satisfies the condition of the particle number conservation:

$$
\sum_{n=1}^{N} n g_{n}=\text { const }=\phi / \mathrm{V}
$$

where $\mathrm{V}$ is the volume of a particle, and $\varphi$ is the total volume fraction of the particles. For the kinetic coefficients $\alpha_{m n}$ we will use the following estimate, suggested in Ref. [8]:

$$
\alpha_{k n}=\int_{S_{a t t r}^{*}} \mathrm{v}_{r}^{*} \mathrm{~d} S
$$

where

$$
\mathrm{v}_{r}^{*}= \begin{cases}\mathrm{v}_{\mathrm{r}}, & \mathrm{v}_{\mathrm{r}} \leq 0 \\ 0, & \mathrm{v}_{\mathrm{r}}>0\end{cases}
$$


and

$$
\begin{aligned}
\mathrm{v}_{\mathrm{r}}= & \frac{\beta_{k n}}{4 \pi \mu_{0}}\left(\frac{m}{\mathrm{~d}}\right)^{2}\left[-\frac{r-\mathrm{d} \cos \theta}{\left(r^{2} \sin ^{2} \theta+(r \cos \theta-\mathrm{d})^{2}\right)^{\frac{3}{2}}}-\frac{r+\mathrm{d}(k+n-1) \cos \theta}{\left(r^{2} \sin ^{2} \theta+(r \cos \theta+\mathrm{d}(k+n-1))^{2}\right)^{\frac{3}{2}}}\right. \\
& \left.+\frac{r-\mathrm{d}(k-1) \cos \theta}{\left(r^{2} \sin ^{2} \theta+(r \cos \theta+\mathrm{d}(k-1))^{2}\right)^{\frac{3}{2}}}+\frac{r-\mathrm{d}(n-1) \cos \theta}{\left(r^{2} \sin ^{2} \theta+(r \cos \theta+\mathrm{d}(n-1))^{2}\right)^{\frac{3}{2}}}\right] .
\end{aligned}
$$

The velocity $v_{r}$ is the product of a chain mobility times the magnetic force of interaction between two chains. This force corresponds to an interaction between equivalent charges located at the extremity of the two interacting chains (see details in Refs. [1,8]).

Here $m$ is the magnetic moment of a particle, estimated below, $d$ the diameter of the particle, $r$ the distance between centers of two particles situated on the nearest extremities of two interacting chains, $\mu_{0}$ the magnetic permeability of vacuum, and $\beta_{k n}$ is a coefficient of mutual hydrodynamical mobility of two chains. In Ref. [8] this parameter has been taken as follows:

$$
\begin{array}{ll}
\beta_{k n}=\left(\beta_{\mathrm{k}}+\beta_{\mathrm{n}}\right) & \beta_{l}=\frac{1}{16 \pi \eta}\left(\chi_{l}+\mathrm{d}^{2} l^{2} \gamma_{l}\right) \\
\chi_{l}=\int_{0}^{\infty} \frac{\mathrm{d} \lambda}{\Delta_{l}(\lambda)} & \gamma_{l}=\int_{0}^{\infty} \frac{\mathrm{d} \lambda}{(l \mathrm{~d}+\lambda) \Delta_{l}(\lambda)} \quad \Delta_{l}(\lambda)=\sqrt{\left(l^{2} \mathrm{~d}^{2}+\lambda\right)\left(\mathrm{d}^{2}+\lambda\right)\left(\mathrm{d}^{2}+\lambda\right)} .
\end{array}
$$

In Eq. (2) we integrate over the surface $S$ of two half spheres with a radius $r$ equal to the mean distance between the chain extremities (see, discussion of this integration in Ref. [8]). These half spheres, located near both extremities of the chain, correspond to areas of attraction between the chains and exclude their lateral aggregation. The simplest estimate for the distance $r$ has the following form [8]:

$$
r \approx R=\mathrm{d} \varphi^{-1 / 3} / 2
$$

It was shown in Ref. [8] that for the 2D case an approximation like the one of Eq. (5) leads to quite reasonable estimates of $g_{n}$ when the particle surface concentration does not exceed one-two per cent.

For a maximal simplification of the calculations we neglect the effect of magnetic interaction between particles on their magnetic moments. Under the assumption of a constant permeability $\mu_{p}$ of the particle, we have for the magnetic moment [9]:

$$
m=\frac{\pi \mu_{0} \mathrm{~d}^{3}}{2} \frac{\mu_{p}-1}{\mu_{p}+2} H
$$

where $\mu_{0}$ is the vacuum permeability.

Assuming that, at the onset of aggregation, all particles are isolated, we have the following initial conditions for Eq. (1)

$$
t=0, \quad g_{n}=\frac{1}{V} \varphi \delta_{n 1} .
$$

Here $\delta_{n 1}$ is the Koneke symbol.

The Eqs. (1)-(7) can be solved numerically.

\section{Computer simulations}

We consider a system of identical magnetic spheres. The magnetic moment of each particle in the computer simulation, like in the analytical model, is given by Eq. (6). In the simulation we take into account magnetic interactions between all particles but ignore hydrodynamical interactions between them.

Neglecting inertia, the equation of motion of the $i$ th particle is:

$$
\frac{\mathrm{d} \mathbf{r}_{i}}{\mathrm{~d} t}=\frac{1}{3 \pi \eta \mathrm{d}}\left(\sum_{j \neq i}\left(\mathbf{F}_{m}^{i, j}+\mathbf{F}_{s t}^{i, j}\right)\right)
$$

Here $\mathbf{r}_{i}$ is the position of the $i$ th particle, $\mathbf{F}_{m}^{i, j}$ and $\mathbf{F}_{s t}^{i, j}$ are the magnetic and sterical forces of interaction between the $i$ th and $j$ th particles, $\eta$ is viscosity of the carrier liquid.

Approximation (8) means that the hydrodynamical mobility of the particle in the chain is the same as the mobility of the single particle. This approximation is frequently used in computer simulations of MRS (see, for example, Refs. [10,11]). Analysis shows that this approximation does not lead to serious quantitative errors. 
In the framework of the dipole-dipole interaction, the radial and tangential components of the magnetic force can be calculated as:

$$
\begin{aligned}
F_{m, r}^{i, j} & =\frac{3 m_{i} m_{j}}{4 \pi \mu_{0}} \frac{1-3 \cos ^{2} \theta}{r_{i, j}^{4}} \\
F_{m, \theta}^{i, j} & =-\frac{3 m_{i} m_{j}}{4 \pi \mu_{0}} \frac{2 \sin \theta \cos \theta}{r_{i, j}^{4}} .
\end{aligned}
$$

Here $r_{i, j}$ is the distance between the particle centers, $m_{i}$ and $m_{j}$ are their magnetic moments, $\theta$ is the angle between the applied field $\mathbf{H}$ and the radius vector $\mathbf{r}_{i j}$.

The short range sterical force between particles preventing their overlapping was applied when the distance between their centers was lower than the diameter $\left(r_{i j} \leq \mathrm{d}\right)$. This force makes the particles leave the region of the interpenetration during one time step. At the same time, the force of magnetic attraction was equated to zero. As soon as the particles separated under the repulsion force $\left(r_{i j}>\mathrm{d}\right)$, we put this force equal to zero and reintroduce the magnetic force.

\section{Results of 2D simulation and the effect of the cell size}

We took advantage of the 2D geometry to explore the long time behavior where asymptotic laws are known for the size distribution and for the growth of the average size of the clusters with time. For those 2D simulations, we used periodical boundary conditions in the direction perpendicular to the field (lateral dimension) to avoid boundaries effects. The dipolar moment of the particles is constant and the magnetic interactions are computed for distance up to the cell size. The simulations were carried for 4 different surface fractions $(3.5 \%, 5.6 \%, 7 \%, 8.9 \%)$ and two different sizes of the box; the lateral dimension (width) is 62.5 particles diameters in all cases and the height is either 62.5 or 31.25 particles diameters.

The average chain length, is $l d$, the mean number $l$ of the particles in the chain by definition can be calculated as:

$$
l(t)=\frac{\sum_{k} k n_{k}(t)}{\sum_{k} n_{k}(t)} .
$$

Here $n_{k}(t)$ is the number of chains containing $k$ particles. The results of the computer simulations have been adjusted by the power law $l(t) \propto t^{\gamma}$. The exponent $\gamma$ allows us to predict the growth of the average chain size with time.

The asymptotic behavior of the size distribution and the exponent $\gamma$ can be deduced from the scaling behavior of the kinetic coefficient $\alpha_{i j}$, which is proportional to the relative velocity $v_{i j}$ of two chains of respective size $i$ and $j$ diameters, times the capture surface: $\alpha_{i j}=v_{i j} S_{i j}$ [12]. The relative velocity $v_{i j}$ is given by the product of the relative mobility, which is proportional to $1 / i+1 / j$, by the force between two aggregates which is proportional to $m^{2} / R_{i j}^{2}$ [1]. The surface $S_{i j}$ being proportional to $R_{i j}^{2}$ in 3D and to $R_{i j}$ in 2D cases respectively, we end up with the following relations for the exponent $\gamma$ [13]:

$$
\begin{aligned}
& \alpha_{i, j} \propto\left(\frac{1}{i}+\frac{1}{j}\right) \text { and } \alpha_{\lambda i, \lambda j}=\lambda^{-1} \alpha_{i, j}=\lambda^{2 \omega} \alpha_{i, j} \Rightarrow \gamma=\frac{1}{1-2 \omega}=\frac{1}{2} \text { in 3D } \\
& \alpha_{i, j} \propto\left(\frac{1}{i}+\frac{1}{j}\right) \frac{1}{R_{i j}} \text { and } \alpha_{\lambda i, \lambda j}=\lambda^{-3 / 2} \alpha_{i, j}=\lambda^{2 \omega} \alpha_{i, j} \Rightarrow \gamma=\frac{1}{1-2 \omega}=\frac{2}{5} \text { in 2D. }
\end{aligned}
$$

Where $\lambda$ and $2 \omega$ are the self similarity multiplier and exponent; the last is equal here to -1 and to $-3 / 2$ in $3 \mathrm{D}$ and $2 \mathrm{D}$ cases respectively.

In this last equation it is assumed that, as in Ref. [2], the average distance $R_{i i}$ between two aggregates of size $i$ is given by $: R_{i i} \propto \mathrm{d}\left(\frac{i}{\phi}\right)^{1 / 2}$. Nevertheless we are not dealing with a hierarchical model where all the chains have the same size at a given moment of time and we are looking for $R_{i j}$ and not $R_{i i}$. An ansatz was to take $R_{i j} \propto(\max (i, j))^{1 / 2}[8]$ which gives also the exponent $2 / 5$ in $2 \mathrm{D}$. It is worth noting that the Doi theory [2] predicts the same exponents, that is to say $1 / 2$ in $3 \mathrm{D}$ and $2 / 5$ in $2 \mathrm{D}$.

In Fig. 1 we have plotted the comparison between the results of the numerical simulation and the result of the analytical equations for the two different sizes of the box and the lowest surface fraction $\phi=3.54 \%$. The dimensionless time $\tau$ is defined as: $\tau=2 \frac{\mu_{0} H^{2}}{\eta}$, where $H$ is the magnetic field in the cell. In our simulations the relation between the dipole $m$ and the field $\mathrm{H}$ is given by Eq. (6) in the limit $\mu_{p} \gg 1$.

The average length of the chains is equal to the average number of particles per chains (10) multiplied by the particle diameter. This is true as long as lateral aggregation of the chains is negligible, since in the absence of Brownian motion the chains of particles are perfectly straight so detecting the length of the clusters is equivalent to detecting the number of particles in the cluster.

The two curves with a staircase shape are the results of numerical simulations. The lower one corresponds to the height of 31 diameters for the simulation box and the upper one to 62 diameters. Each curve is the average of three simulations.

The continuous solid lines are the respective results of the kinetic equation (1). It appears that this equation reproduces quite well the numerical simulation except for the smallest sizes. This is understandable since the model of interactions between equivalent charges at the extremity of the chains (Eq. (3)) is rather poor for the shortest chains. 


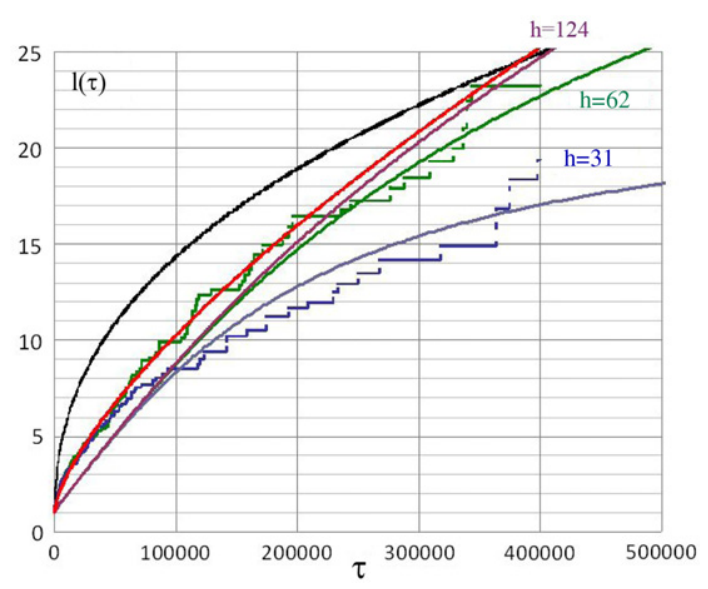

Fig. 1. Average length of chains (in number of particles) vs. the dimensionless time for a surface fraction $\phi=3.54 \%$. Blue and green stands respectively for a box of height $h=31$ and 62 of the particle diameters respectively. The staircase shape is the numerical simulation result and the continuous line is the solution of Eq. (1) The purple solid line is the solution of Eq. (1) for a box of height 124 diameters. The upper black line is the 2D version of See and Doi theory [2]. Finally the tiny red curve is the fit of the simulation $h=62$ by a power law for $l<15$.

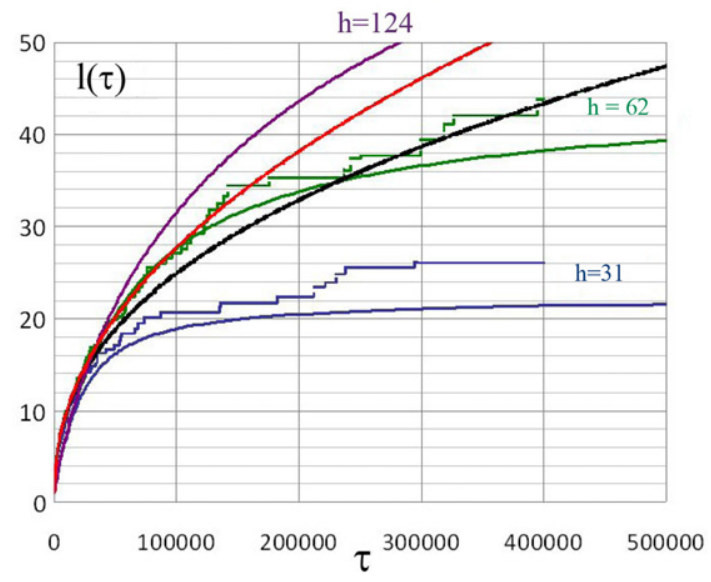

Fig. 2. Average dimensionless length of the chains vs. the dimensionless time for the particle surface fraction $\phi=8.91 \%$. Blue and green lines stand respectively for a box of 31 particle diameter and 62 diameters. The staircase shape is the numerical simulation result and the solid line is the solution of Eq. (1). The purple solid line is the solution of Eq. (1) for a box of height 124 diameters. The black solid line is the 2D version of the model [2]. Finally, the tiny red curve is the fit of the simulation with $h=62$ by a power law for $l(\tau)<20$.

The growth of the average size with time is rather quickly sensitive to the size of the box; for instance the blue curves corresponding to a box of 31 diameters begin to diverge from the green ones corresponding to 62 diameters at approximately 7-8 diameters, that is to say when the average size is about $25 \%$ of the box height. The same remark holds when we compare the result of Eq. (1) between 124 and 62 diameters: it begins at about 15 diameters which is still $25 \%$ of the size of the smallest box. It corresponds to the situation where, in the smaller box, the size of the largest aggregates reach between 1/3 and 1/2 of the size of the box, thus limiting the possibility of end to end aggregation. If we are looking for the power law behavior, we have to take into account that it should hold only for infinite system, so it can only be deduced from a fit on the first part of the curve; for instance for $\mathrm{n}<15$ concerning the simulation with a height of 62 diameters. The result is presented as the red curve in Fig. 1 and corresponds to the equation: $l(\tau)=1+0.003 \tau^{0.696}$. Now we can use the result of Eq. (1) for a height of 124 diameters and fit the curve for $n(t)<30$; in this case the fit gives $n(\tau)=1+0.00076 \tau^{0.8}$. We see that the exponent of the power law can depend of the size of the box. In any event we are far from the exponent $2 / 5$ that is predicted by the standard theory. This is not completely surprising because this exponent is only valid if the kinetic coefficient presents an homothetic relation versus the size of the chains, which is satisfied if the length of the chains is large enough to keep only the first term in Eq. (3).

The upper curve in Fig. 1 is the one predicted by the hierarchical model [2] which follows the law:

$l(\tau)=\phi^{3 / 5} \frac{\left(2^{5 / 2}-1\right)}{4^{2 / 5}} \tau^{2 / 5}$. This theory clearly strongly overestimates the initial growth of the chains.

For the higher surface fraction $(\phi=8.91 \%)$, the results of the chain average size versus time are presented in Fig. 2 . 


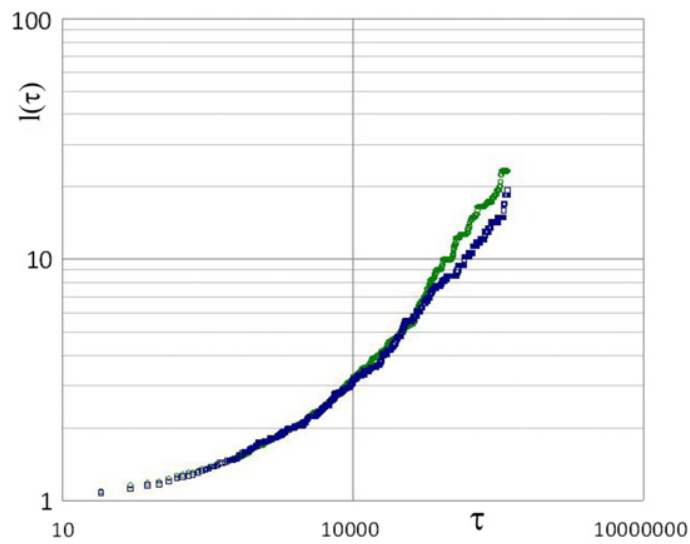

Fig. 3. Log-Log plot of the average size of clusters for the particle surface $\phi=3.54 \%$ and the two heights $h=31$ (blue square), $h=62$ (green dot).

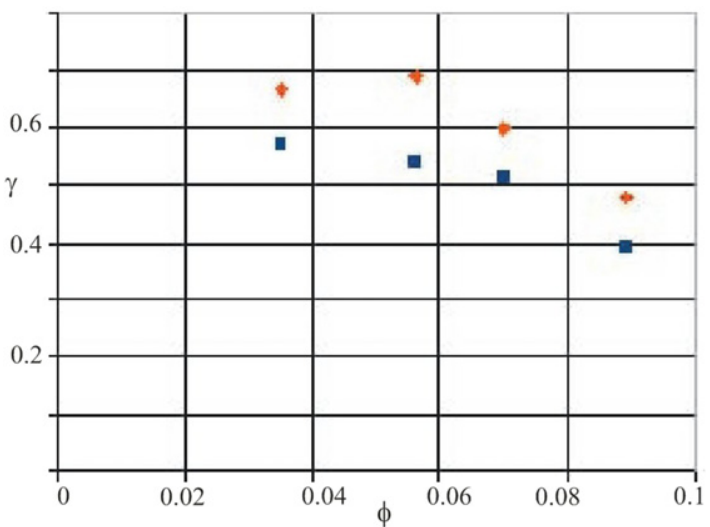

Fig. 4. Long time exponent of the law $l(\tau)=A \tau^{\gamma}$ obtained by numerical simulation vs. the surface fraction; red lozenge $h=62$; blue square $h=31$.

Increasing the concentration enhances the dependence of the results on the box height-the difference between the curves for the heights $h=31$ and $h=62$ in Fig. 2 is more significant than the one in Fig. 1. Agreement between the computer simulations and the solution of the Eq. (1) is rather good.

However, the decrease of the chain growth rate is faster in the kinetic equation than in the simulation; this is due to the lateral aggregation of chains which is not taken into account in the Eq. (1).

The lateral aggregation contributes to increase the number of the largest chains and actually is responsible for the percolation of chains between the two sides of the box. This aspect is discussed in the next section devoted to the 3D simulations and will be studied in more details in a future work. Coming back to the power law for $l(\tau)$, when the fit is realized for $l<20$, where the simulation begins to diverge from the analytical result with $h=124$, we obtain: $l(\tau)=1+0.108 \tau^{0.48}$ showing a large decrease of the exponent when the volume fraction increases. Nevertheless this result is still dependant on the box height, and if the analytical result for $h=124$ is adjusted by a power law with $l<30$, we find $l(\tau)=1+0.018 \tau^{0.65}$. Finally the hierarchical model (solid black line) with a power $2 / 5$ is rather close to the simulation result for $h=62$ but it does not mean a lot. Actually, since this model does not take into account the box size its prediction should be above the analytical result corresponding to $h=124$.

Previous values of the parameters of the $l(\tau)$ power law were obtained fitting the data from $\tau=0$ to a maximum time such that the average size is approximately $1 / 4$ of the box size. It is more usual to calculate the power law exponent on the linear part of a Log-Log plot. In the Fig. 3 we show the Log-Log plot of the results presented in Fig. 1 on a longer time scale.

The linear part corresponding to a power law begins approximately above $l=5$. The corresponding exponents are plotted in Fig. 4 for the two heights $h=31$ and $h=62$ particles diameter. Some results of fit of the parameters $A$ and $\gamma$ of the law $l(\tau)=A \tau^{\gamma}$ are given in the Table 1 .

We see here a decrease of the exponent $\gamma$ with the volume fraction. This decrease can be interpreted by the formation of double chains or clusters of chains formed by lateral aggregation. In this case the length of the chain is growing slower than the number of particles in the chain, which explains this decrease with the volume fraction. The other point is that the largest box (red stars) shows a larger exponent than the smallest one (blue square). As discussed previously the influence of the size of the box, which is to decrease the size of the chains, is felt as soon as the average length is above one quarter of the box size and explains this difference of exponent. Nevertheless, for applications like pressure sensors based on the 


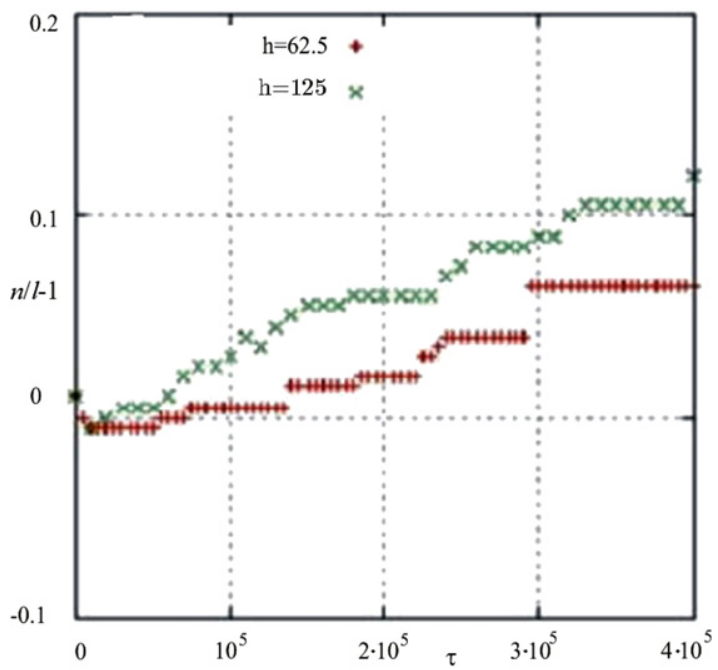

Fig. 5. Average overlap between two chains versus time; $n / l$ is the number of particles inside a cluster divided by its length and averaged on all cluster sizes.

Table 1

Values of the parameter A and $\gamma$ corresponding to the Fig. 4.

\begin{tabular}{lllll}
\hline$\phi$ & $\gamma(h=31)$ & $\mathbf{A}(h=31)$ & $\gamma(h=62)$ & $\mathbf{A}(h=31)$ \\
\hline 0.035 & 0.57 & 0.0105 & 0.67 & 0.004 \\
0.056 & 0.54 & 0.0305 & 0.69 & 0.007 \\
0.07 & 0.51 & 0.054 & 0.60 & 0.0235 \\
0.089 & 0.39 & 0.24 & 0.48 & 0.105 \\
\hline
\end{tabular}

conductivity of chains of particles [14] the time needed to obtain a percolation between the opposite sides of the cell is the quantity of interest and it is worth noting that at time $\tau=40000$ the average chain length respective to the size of the box is $l / h=0.69$ for $h=61$ and $l / h=0.84$ for $h=31$. It means that, as expected intuitively, the percolation time decreases with the size of the box. Nevertheless this decrease is not as strong as expected because a chain of size $k d$ can only connect end by end with a chain of length $j d<h d-k d$ and side by side aggregation will in practice play the preponderant role for the realization of the percolation between the two sides of the cell. This side by side aggregation can be quantified by dividing the number of particles inside the aggregate by its length. When this number is larger than unity it means that we have lateral aggregation and the difference with unity measures the proportion of particles belonging to double chains. This is plotted versus time in Fig. 5 for the highest area fraction $\phi=8.9 \%$.

Note that the situation for an intermediate area fraction $\phi=4.5 \%$ was completely different since we did not observe any overlap. We see clearly an increase with time of this lateral aggregation and it is more pronounced for the larger cell. This is due to the fact that a lateral aggregation between two chains is dictated by the interaction between equivalent charges at their extremities, so they need to be shifted by at least one half of their length to attract each other. This situation is less and less possible for small heights of the cell where the walls block the possibility to have this shift.

\section{Results of 3D simulations and comparison with analytical model}

We have used about 6000-12000 particles in the computer simulations and solved numerically Eq. (8) for all particles. At the onset of the simulations a random particle distribution has been created. The time step has been chosen so that the particle displacement for a given step would not exceed $\mathrm{d} / 20$. The particles are situated in a parallelepiped region with boundaries impenetrable for the particles. Because of the low concentration of the particles, the demagnetizing shape effects of the gap are weak. It allows us neglect them, assuming that the field in the parallelepiped equals to the applied field.

Some results of our simulations for the systems with small concentrations of the particles are illustrated in Figs. 6 and 7. The viscosity of the carrier liquid, size of the particles and the magnetic field correspond to experiments made with nickel particles that we are currently doing in order to test this model.

Results of the analytical model and computer simulations are in reasonable agreement. Therefore this analytical model can be applied, at least for a first estimation of the chain formation in magnetic suspension with small concentration when only linear aggregates appear in the suspension. As for 2D case we have fitted our results for the average size of the chains (in diameter or equivalently in number of particles) versus time $l=1+\mathrm{Ct}^{\gamma}$ (here the time is in second and not in the reduced units). As in the $2 \mathrm{D}$ case the exponent $\gamma$ decreases when the particles volume fraction $\varphi$ increases above $1 \%$ and more strongly above $1.5 \%$. Like in the case of the $2 \mathrm{D}$ aggregation the decrease of $\gamma$ with $\varphi$ can be explained by the lateral aggregation of the chains. (See Table 2.) 


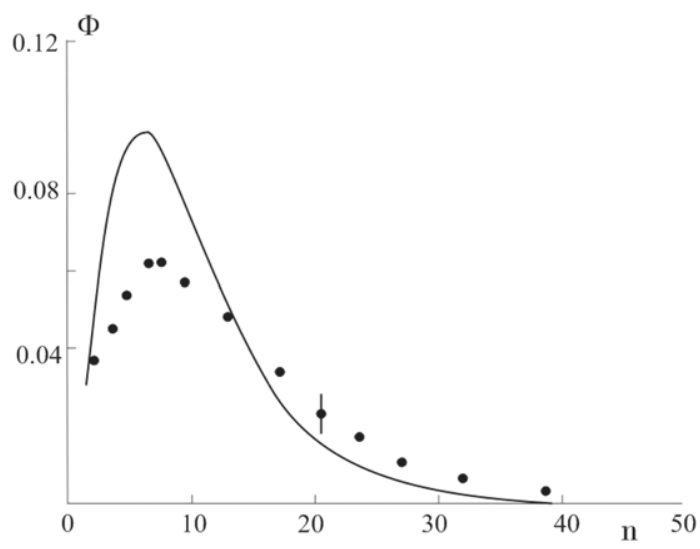

Fig. 6. Relative number $\Phi=\frac{n g_{n} V}{\varphi}$ of particles in the chains vs. number $n$ of particles in the chains. Volume of the particles is $\varphi=0.014$; dimensionless thickness of the gap $h=50$; viscosity of the carrier liquid is $20 \mathrm{~Pa} \mathrm{sec}$. Magnetic induction is $16.5 \mathrm{mT}$. Black line-calculations using the analytical model (1); dots-results of computer modeling, averaged over four simulations. The error bar is shown. Elapsed time after the field was switched on: $t=450 \mathrm{~s}$.

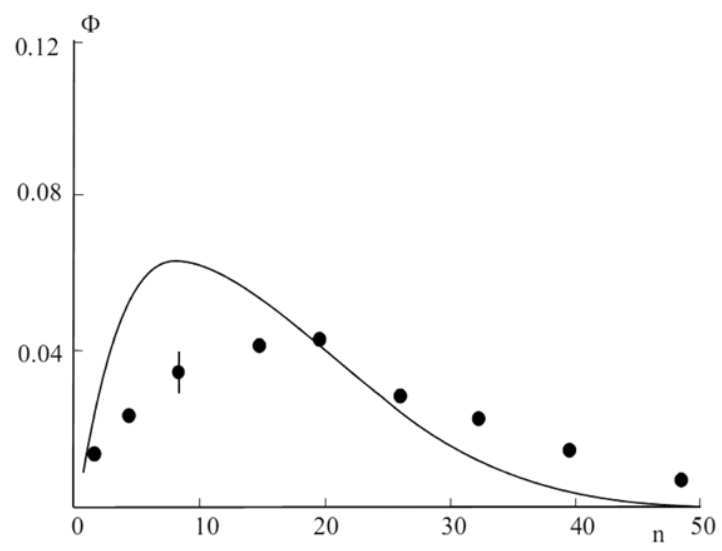

Fig. 7. Same as in Fig. 6 for time $t=900 \mathrm{~s}$.

Table 2

The parameters of the law $l=1+C t^{\gamma}$ for a cubic box with the sizes $50 \times 50 \times 50$ of the particle diameter.

\begin{tabular}{lll}
\hline$\varphi(\%)$ & $\gamma$ & $C$ \\
\hline 0.5 & 0.753814 & 0.0223627 \\
0.75 & 0.807047 & 0.0267223 \\
1 & 0.774925 & 0.0491677 \\
1.25 & 0.728957 & 0.0835969 \\
1.5 & 0.707 & 0.115781 \\
2 & 0.631208 & 0.252217 \\
\hline
\end{tabular}

\section{Formation of thick clusters}

Actually when the volume concentration of the particles $\varphi$ does not exceed $1.5 \%$, almost only linear chains are observed in our simulations. Increasing the particle concentration leads to the appearance of bulk clusters. Some of these clusters are shown in Figs. 8 and 9.

Fig. 10 illustrates the evolution of the internal structures in the plane of the gap, i.e. perpendicular to the applied field direction.

In this plane the projection the structures have a labyrinth-like shape. This feature of the dense structures in systems of polar non Brownian particles has been noted in computer simulations [10]. Experimentally the irregular branch and labyrinth-like structures in thin layers of MRS have been observed, for example, in Ref. [15]. It should be noted that in layers of suspensions of magnetic nano-sized particles (ferrofluids) subjected to intensive Brownian motion, the structures, that appear when a field is applied, are usually rather cylindrical, with quite regular shape (see photos, for example, in Ref. [16]). The principal difference between structures in ferrofluids and MRS is that in the ferrofluids the particles, due to the Brownian 
a

b

C

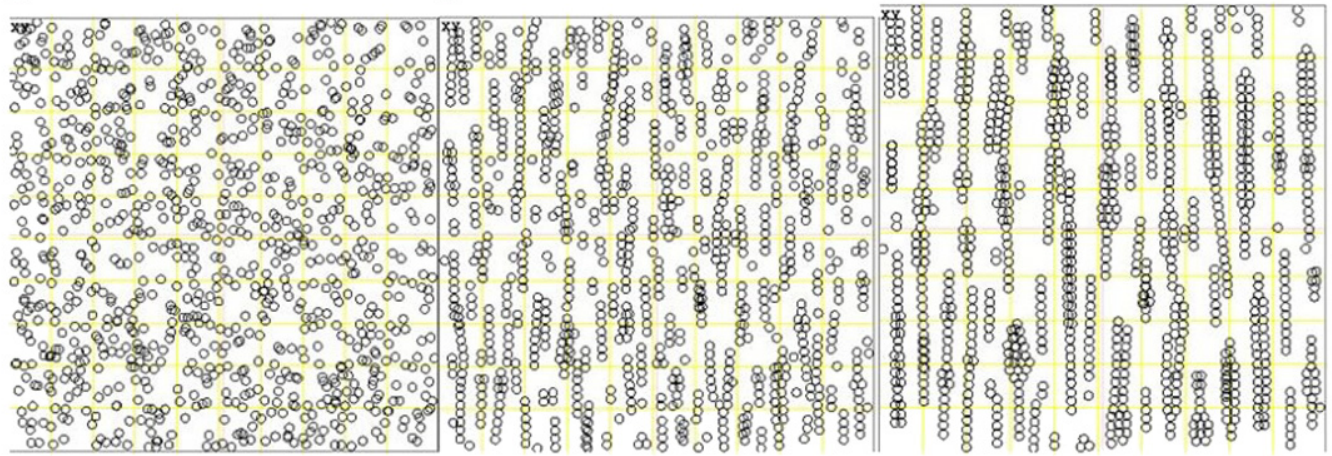

Fig. 8. Screenshot of the system of the particles in a gap which thickness $h=400$. Magnetic field is applied in vertical direction, in the plane of the Figure. Volume concentration of particles $\varphi=0.05$; time after the field was switched on : $\mathrm{a}-t=4 \mathrm{~s} ; \mathrm{b}-t=10 \mathrm{~s} ; \mathrm{c}-t=50 \mathrm{~s}$.

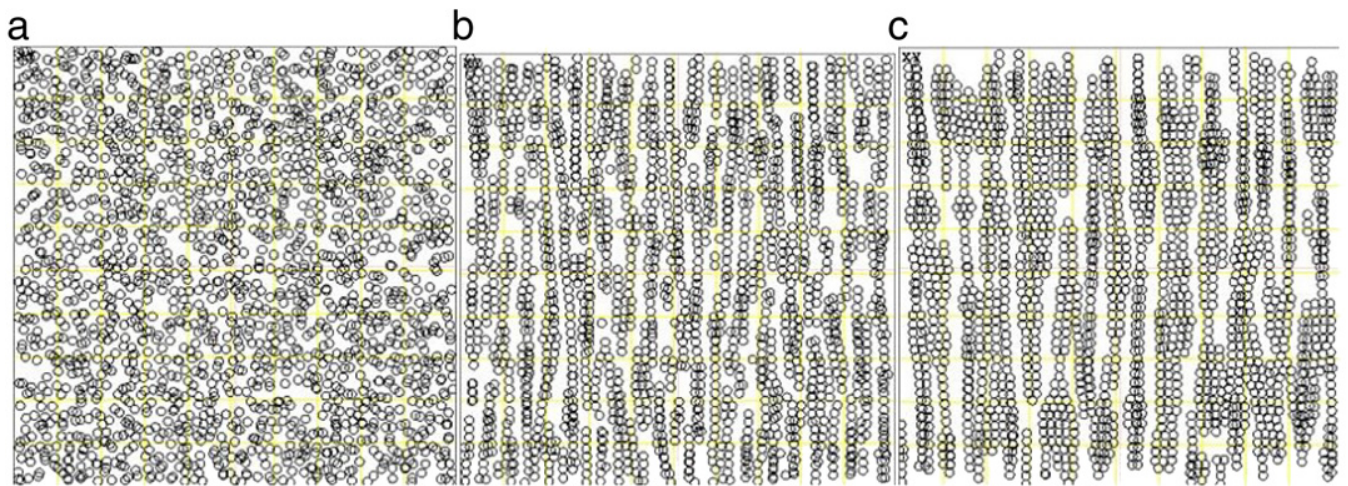

Fig. 9. Same as in Fig. 8 when the particle volume concentration $\varphi=0.1$.

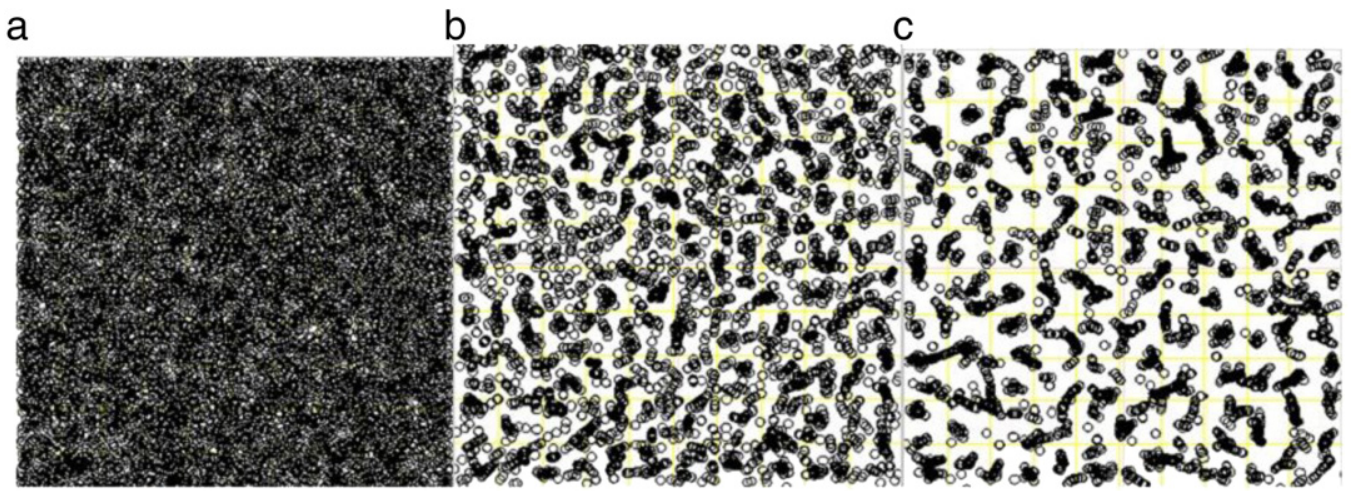

Fig. 10. Evolution of internal structures in suspension with volume fraction of particles $\varphi=0.05$. View in a plane perpendicular to the field. Thickness of the gap $h=400$; (a) at the moment when the field is switched on; (b) after $t=50 \mathrm{~s}$; (c) after $t=200 \mathrm{~s}$.

motion, can achieve thermodynamically equilibrium disposition. In MRSs the non Brownian particles are "frozen" in some local energy minimums.

We have also investigated the effect of the gap thickness on the features of the internal structures. Our observations show that, like in the 2D case, if the gap thickness is relatively small, only linear chains take place in the system. Increase of this size of the gap leads to appearance of the bulk branched aggregates.

\section{Summary}

A model for the kinetics of chain formation in a magnetorheological suspension confined between two walls is presented. Mathematically this model is based on the system of the Smoluchowski equations for the distribution function of the number 
of particles in the chain. This model well reproduces the results of computer simulation of the aggregates growth in confined cells as long as the volume fraction of particles remains low enough to avoid lateral aggregation between the chains. Analysis shows that the dependence of the mean size of the chain on time can be fitted by a power law. The parameters of this power function are sensitive to the gap size and to the particle concentration. The lateral aggregation of chains, which is significant when the particle concentration is high enough, provokes a decrease of the exponent of the power law. Furthermore when the particle volume fraction exceeds $=$ approximately $5 \%$, dense aggregates with labyrinthine structures are observed in the simulations.

In the model as well as in computer simulations the particles are not supposed to be attracted by the walls of the cell. However in many experimental situations this attraction takes place. We are now planning to study aggregation in magnetic suspensions when the particles are attracted to the cell walls.

\section{Acknowledgments}

This work has been supported by the grants of the Russian Fund of Fundamental Investigations, NN 10-01-96002-Ural, 10-02-96001- Ural, 10-02-00034, 12-01-00132; Federal Goal Program of Russian, agreement No. 14.A18.21.0867, Scientific Program of Russian Ministry of Education, pr. 2.1267.2011 and by the European Commission within the DYNXPERTS project (ref. FP7-2010-NMP-ICT-FoF-260073).

\section{References}

[1] G. Bossis, O. Volkova, S. Lacis, A. Meunier, in: S. Odenbach (Ed.), In Ferrofluids Magnetically Controllable Fluids and Their Applications, Springer, 2002.

[2] H. See, M. Doi, J. Phys. Soc. Japan 60 (1991) 2778.

[3] E. Climent, M.R. Maxey, G.E. Karniadakis, Langmuir 20 (2004) 507.

[4] M.C. Miguel, R.P. Satorras, Phys. Rev. E 59 (1999) 826.

[5] P. Dominguez-Garcia, S. Melle, J.M. Pastor, M.A. Rubio, Phys. Rev. E 76 (2007) 051403.

[6] Z. Mimouni, J.A.D Wattis, Physica A 388 (2009) 1067.

[7] G. Bossis, C. Métayer, A. Zubarev, Phys. Rev. E 76 (2007) 041401.

[8] G. Bossis, L. Iskakova, V. Kostenko, A. Zubarev, Physica A 390 (2010) 2655.

[9] L.D. Landau, E.M. Lifshitz, Electrodynamics of Continuum Media, Pergamon Press, London, 1960.

[10] M. Mohebi, N. Jamasbi, Phys. Rev. E. 54 (1996) 5407.

[11] Z-J. Tan, X-W. Zou, W-B. Zhang, Z-Z. Jin, Phys. Rev. E. 59 (1999) 3177.

[12] R. Botet, R. Jullien, J. Phys. A: Math. Gen. 17 (1984) 2517.

[13] R. Jullien, R. Botet, Aggregation and Fractal Aggregates, World Scientific, 1987.

[14] N. Kchit, G. Bossis, J. Phys. D: Appl. Phys. 42 (2009) 105505.

[15] J.C. Bacri, D. Salin, J. Magn. Magn. Matter 9 (1983) 48; E. Blums, A. Cebers, M. Majorov, Magnetic Fluids, de Gruyter, Berlin, 1997.

[16] Modesto T. Lopez-Lopez, Juan D.G. Duran, Laura Rodriguez-Arco, Andrey Zubarev, Larisa Iskakova, J. Appl. Phys. 108 (2010) 083503. 